\title{
NUDT expression is predictive of prognosis in patients with clear cell renal cell carcinoma
}

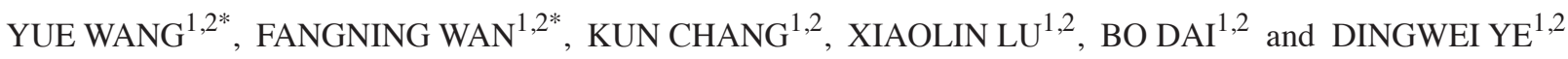 \\ ${ }^{1}$ Department of Urology, Fudan University Shanghai Cancer Center, ${ }^{2}$ Department of Oncology, \\ Shanghai Medical College, Fudan University, Shanghai 20032, P.R. China
}

Received February 21, 2016; Accepted July 27, 2017

DOI: $10.3892 / \mathrm{ol} .2017 .6997$

\begin{abstract}
The nudix hydroxylase (NUDT) family of genes may have notable roles in cancer growth and metastasis. The present study aimed to determine the prognostic ability of NUDT genes in clear cell renal cell carcinoma (ccRCC). Data from 509 patients with ccRCC was obtained from The Cancer Genome Atlas (TCGA) database and 192 patient samples from Fudan University Shanghai Cancer Center (FUSCC) were analyzed in the present study. The expression profile of NUDT gene family members in the TCGA cohort was obtained from the TCGA RNA sequencing database. Pathological characteristics, including age, sex, tumor size, tumor grade, stage, laterality and overall survival were collected. Cox proportional hazards regression model and Kaplan-Meier survival analysis were performed to assess the associations between pathological characteristics and expression levels of NUDT family genes. NUDT family genes that exhibited associations with overall survival (OS) were further validated in the FUSCC cohort. In the TCGA cohort, Cox proportional hazards analysis found that NUDT5 [hazards ratio $(\mathrm{HR})=1.676 ; 95 \%$ confidence interval (CI), 1.097-2.559] and NUDT17 (HR=1.375; 95\% CI, 1.092-1.732) were predictive of ccRCC prognosis. Further analysis revealed that low NUDT5 $(\mathrm{P}<0.0001)$ and NUDT17 $(\mathrm{P}<0.0001)$ expression were associated with poorer OS rates in the TCGA cohort. In the FUSCC cohort, low NUDT5 expression was also associated with poor OS rates $(\mathrm{P}=0.0116)$, and tumor grade was a factor that influenced the expression level of NUDT5 $(\mathrm{P}=0.016)$.
\end{abstract}

Correspondence to: Dr Bo Dai or Dr Dingwei Ye, Department of Urology, Fudan University Shanghai Cancer Center, 270 DongAn Road, Shanghai 20032, P.R. China

E-mail: bodai1978@126.com

E-mail: dwyeli@163.com

*Contributed equally

Key words: clear cell renal cell carcinoma, nudix hydroxylase family, prognosis

\section{Introduction}

Renal cell carcinoma (RCC) is responsible for about $3 \%$ of all malignancies in adults, and 250,000 new cases of kidney cancer are diagnosed each year worldwide (1). At present, clear cell RCC (ccRCC) is the most common form of adult kidney cancer, representing a diverse set of neoplasms with unique genetic and histological features $(2,3)$. Despite developments in diagnosis and treatment strategies of RCC during the past few years, one-third of patients present with metastatic disease at diagnosis (2). Furthermore, 20-40\% of RCC patient that undergo surgical nephrectomy will develop metastasis, meaning poor prognosis. Prognostic factors for RCC include histological subtype, nuclear grade, tumor size, local extent of the tumor and evidence of metastatic disease at presentation $(4,5)$. Although a number of targeted drugs have emerged in recent years, the overall survival times of patients with metastatic kidney cancer remain short (6). ccRCC is generally resistant to standard chemotherapy and radiotherapy. Previous studies have revealed that the 5-year survival rate of patients with metastatic RCC is $<10 \%(7,8)$. Therefore, to increase understanding of ccRCC prognosis and to develop novel biological therapies, it is necessary to identify molecular markers that have the potential to improve patient outcomes and provide novel molecular targets for adjuvant therapies.

Nudix hydroxylases (NUDTs) are a superfamily of $\mathrm{Mg}^{2+}$-coupling enzymes found in viruses, archaea, bacteria and eukaryotes, and catalyze the hydrolysis of nucleoside diphosphates associated with other moieties, X (any moiety) (9). There are two components to the Nudix hydroxylases family: the so-called Nudix hydroxylases fold of a $\beta$-sheet with $\alpha$-helices on each side and the Nudix hydroxylases motif which contains catalytic and metal-binding amino acids. The Nudix hydroxylases motif is GXXXXXEXXXXXXXREUXEEXGU where $\mathrm{U}$ is isoleucine, leucine or valine, and $\mathrm{X}$ is any amino acid (9). All NUDT family members are characterized by a highly conserved 23-residue sequence motif, the Nudix box, and are housecleaning enzymes $(10,11)$. NUDT family enzymes can activate a phosphodiester bond through the $\mathrm{Mg}^{2+}$-assisted nucleophilic attack of a water molecule by a basic residue. The typical NUDT reaction releases products such as N-methyl-2-pyrrolidone, phosphate, or pyrophosphate $(12,13)$.

The human genome has 24 NUDT hydrolase genes and $>5$ pseudogenes, several of which encode more than one variant. 
Expression of 17 of the 19 studied NUDT genes is strongly induced upon entry into stationary phase, which suggests a possible involvement in metabolic reprogramming $(14,15)$. Additionally, numerous site-directed mutagenesis studies have highlighted the importance of individual residues in the Nudix motif for catalysis. However, little is known about the NUDT family in the field of renal cancer.

\section{Materials and methods}

NUDT expression data. Information on the expression of NUDTs and clinical data of the Cancer Genome Atlas (TCGA) database were obtained from the Cancer Genomics Browser of University of California Santa Cruz (https://genome-cancer. ucsc.edu/). A total of 24 members (NUDT1, NUDT2, NUDT3, NUDT4, NUDT5, NUDT6, NUDT7, NUDT8, NUDT9, NUDT9P1, NUDT10, NUDT11, NUDT12, NUDT13, NUDT14, NUDT15, NUDT16, NUDT16P1, NUDT16L1, NUDT17, NUDT18, NUDT19, NUDT21 and NUDT22) of the NUDT family are included in the database. In total, 509 patients (median age, 61 years; range, 26-90 years) with primary ccRCC tumors from with detailed NUDT expression data were chosen from the updated TCGA database according to parameters defined in a previous study (16). Only patients with fully characterized tumors, intact overall survival (OS) data, complete RNAseq information and without pretreatment were included. Clinicopathological characteristics, including age, sex, tumor size, Tumor-Node-Metastasis (TNM) stage (1), tumor grade, laterality, hemoglobin level, white blood cell level, platelet level and overall survival were collected. Follow-up of the patients was completed with a median length of 1,063 days. In total, 347 patients succumbed during the follow-up.

Patient enrollment. For the Fudan University Shanghai Cancer Center (FUSCC) cohort, 192 patients with ccRCC (median age, 55.5; range, 17-84 years) who underwent radical nephrectomy (RN) or nephron sparing nephrectomy (NSS) between February 2007 and November 2011 were retrospectively enrolled. All the tissue samples were collected during surgeries and stored at $-70^{\circ} \mathrm{C}$ in the tissue bank of FUSCC. The pathological subtypes were confirmed by experienced pathologists. Clinicopathological characteristics, including age, sex and tumor size are summarized in Table I. The present study was approved by the Ethics Committee of Fudan University (Shanghai, China). Patient tissues were used to investigate the expression of genes that were thought to potentially be associated with the prognosis of patients with ccRCC. All the patients in the present study provided signed the informed consent for the publication of their data. All patients provided written informed consent to their inclusion in the study.

Reverse transcription-quantitative polymerase chain reaction $(R T-q P C R)$. In the FUSCC cohort, total RNA was isolated from 192 ccRCC samples using TRIzol reagent (Invitrogen; Thermo Fisher Scientific, Inc., Waltham, MA, USA). The PrimeScript RT Reagent kit (K1622; Thermo Fisher Scientific, Inc., Waltham, MA, USA) was used to synthesize first-strand cDNA from total RNA. Next, Synergy Brands (SYBR)-Green real-time PCR assays (Thermo Fisher Scientific, Inc.) were performed using an ABI 7900HT Thermocycler (Applied
Biosystems; Thermo Fisher Scientific, Inc.). The expression level of RNA was normalized, using relative quantification, to the level of $\beta$-actin (17). The primers for qPCR analysis were synthesized by Sangon Biotech Co., Ltd. (Shanghai, China), the sequences of which are shown as follows: NUDT5 forward, 5'-GGACTGACGCATCTGACTGT-3' and reverse, 5'-ACAGCCGACACACACATACC-3'; NUDT9P1 forward, 5'-AGGCTGTGAACTACCGTGATG-3' and reverse, 5'-AGA GGCTGGCATAAAGCTCA-3'; NUDT16 forward, 5'-TCT CTCCCCCAAGAAAGCATC-3' and reverse, 5'-CCAAGG CTCACACCTCACTA-3'; NUDT17 forward, 5'-CCAACC ATGGCAGAGGACAA-3' and reverse, 5'-CTTTCCTGCTTT CCCCCGT-3'; and $\beta$-actin forward, 5'-AGCGAGCATCCC CCAAAGTT-3' and reverse, 5'-GGGCACGAAGGCTCATCA TT-3'.

TNM stage, tumor grade, and other information were obtained from the electronic records of the patients. Patients were regularly followed up on the telephone or in the clinic every 3 months. Events, including tumor recurrence, progression, metastasis and death, were recorded.

Statistical analysis. Disease-free survival was defined as time from the date of diagnosis to the date of first recurrence or mortality. OS was calculated from the date of diagnosis to the date of death or of the last follow-up. Patients without events or death were recorded as censored at the time of last follow-up. Stata 12.0 software (StataCorp LP, College Station, TX, USA) was used to perform statistical analysis. Survival curves were constructed using the Kaplan-Meier method, with log-rank tests used to assess the differences between the groups. Adjusted odds ratios (ORs) with $95 \%$ confidence intervals (CIs) were calculated using the Cox proportional hazards model. Univariate and multivariate Cox Proportional hazards of NUDT family members expression and OS for patients with ccRCC in the TCGA cohort were analyzed. $\mathrm{P}<0.05$ was considered to indicate a statistically significant difference. Genes that were associated with OS were studied further. Multivariate logistic regression was used to further study factors that could affect the expression of NUDTs. Student's t-test or Wilcoxon signed-rank test were performed in 70 couples of paired patients to assess the different expression of NUDT family genes between patients with ccRCC and healthy individuals. A t-test was applied when the test statistic would follow a normal distribution, if not, Wilcoxon signed-rank test was applied.

\section{Results}

Clinical characteristics of patients with ccRCC in TCGA and FUSCC cohort. In the TCGA cohort, the median age of the 509 patients with ccRCC was 61, ranging between 26 and 90 years old. Of these patients, $328(64 \%)$ were male and $181(36 \%)$ were female. Tumor size, TNM stage, tumor grade, laterality, hemoglobin level, white blood cell level and platelet level are shown in Table I. The median follow-up time of this cohort was 79.5 months.

In the FUSCC cohort, the median age of these 192 patients with ccRCC was 55.5, ranging from 17 to 84 years old; 131 $(68.2 \%)$ were male patients and $61(31.8 \%)$ were female patients. Tumor size, tumor grade, TNM stage, and tumor 
Table I. Expression of the nudix hydroxylase family in 70 couples of paired patients in the TCGA cohort.

\begin{tabular}{|c|c|c|}
\hline Variable & TCGA cohort, n (\%) & FUSCC cohort, $\mathrm{n}(\%)$ \\
\hline Total patients & 525 & 192 \\
\hline \multicolumn{3}{|l|}{ Sex } \\
\hline Male & $341(64.95)$ & $131(68.23)$ \\
\hline Female & $184(35.05)$ & $61(31.77)$ \\
\hline \multicolumn{3}{|l|}{ Grade } \\
\hline $1 / 2$ & $240(45.71)$ & $79(41.15)$ \\
\hline $3 / 4$ & $202(38.48)$ & $113(58.85)$ \\
\hline Gx & $8(1.52)$ & $0(0.00)$ \\
\hline \multicolumn{3}{|l|}{ pT } \\
\hline $\mathrm{T} 1$ & $267(50.86)$ & $129(67.19)$ \\
\hline $\mathrm{T} 2$ & $68(12.95)$ & $29(15.10)$ \\
\hline $\mathrm{T} 3$ & $179(34.10)$ & $27(14.06)$ \\
\hline $\mathrm{T} 4$ & $11(2.10)$ & $7(3.64)$ \\
\hline \multicolumn{3}{|l|}{$\mathrm{N}$} \\
\hline N0 & $271(51.62)$ & $181(94.27)$ \\
\hline N1 & $17(3.24)$ & $4(2.08)$ \\
\hline $\mathrm{Nx}$ & $237(45.14)$ & $7(3.64)$ \\
\hline \multicolumn{3}{|l|}{ M } \\
\hline M0 & $421(80.19)$ & $184(95.80)$ \\
\hline M1 & $79(15.05)$ & $7(3.60)$ \\
\hline $\mathrm{Mx}$ & $25(4.76)$ & $1(0.50)$ \\
\hline \multicolumn{3}{|l|}{ Stage } \\
\hline I & $262(49.90)$ & $130(67.71)$ \\
\hline II & $56(10.67)$ & $30(15.62)$ \\
\hline III & $126(24.00)$ & $23(11.98)$ \\
\hline IV & $81(15.43)$ & $9(4.69)$ \\
\hline \multicolumn{3}{|l|}{ Laterality } \\
\hline Left & $247(47.05)$ & $90(46.87)$ \\
\hline Right & $277(52.76)$ & $94(48.95)$ \\
\hline Bilateral & $1(0.19)$ & $8(41.67)$ \\
\hline
\end{tabular}

TGCA, The Cancer Genome Atlas; FUSCC, Fudan University Shanghai Cancer Center; T, Tumor; N, Node; M, Metastasis.

position are shown in Table I. The median follow-up time of this cohort was 47.1 months; 47 patients succumbed during follow-up.

NUDT5, NUDT9P1, NUDT16 and NUDT17 expression were independent prognostic factors for OS in the TCGA cohort. In univariate Cox proportion hazard ratio analysis, age, tumor stage, metastasis, tumor stage, Fuhrman grade (All subsequent mentions of grade are referring to Fuhrman grade), hemoglobin level, white blood cell and platelet count, NUDT1, NUDT3, NUDT4, NUDT5, NUDT6, NUDT7, NUDT9P1, NUDT10, NUDT11, NUDT12, NUDT16, NUDT17, NUDT19, NUDT21 and NUDT22 expression were significantly associated with prognosis in terms of OS of patients with ccRCC in the TCGA cohort $(\mathrm{P}<0.05$; Table II). Multivariate Cox analysis, following adjustment for all the potential prognostic factors, which included age, tumor stage, Fuhrman score, laterality, white blood cell count, blood platelet count, hemoglobin content, NUDT1, NUDT3, NUDT4, NUDT5, NUDT6, NUDT7, NUDT9P1, NUDT10, NUDT11, NUDT12, NUDT16, NUDT17, NUDT19, NUDT21 and NUDT22, indicated that age $(\mathrm{HR}=1.037 ; 95 \% \mathrm{CI}, 1.020-1.053 ; \mathrm{P}<0.0001)$, stage $(\mathrm{HR}=1.602$; 95\% CI, 1.317-1.950; $\mathrm{P}<0.0001)$, laterality $(\mathrm{HR}=0.664 ; 95 \%$ CI, 0.467-0.944; $\mathrm{P}=0.023)$, NUDT5 (HR=1.676; 95\% CI, 1.097-2.559; $\mathrm{P}=0.017)$, NUDT9P1 $(\mathrm{HR}=1.512 ; 95 \% \mathrm{CI}$, 1.143-2.000; $\mathrm{P}=0.004)$, NUDT16 $(\mathrm{HR}=0.692 ; 95 \% \mathrm{CI}$, 0.486-0.985; $\mathrm{P}=0.041)$ and NUDT17 $(\mathrm{HR}=1.375 ; 95 \% \mathrm{CI}$, $1.092-1.731 ; \mathrm{P}=0.007)$ were the only independent predictors of OS (all $\mathrm{P}<0.01)$ (Table II).

High expression of NUDT5 and NUDT17 were associated with better prognosis and longer OS in the TCGA cohort. Further study of NUDT5, NUDT9P1, NUDT16 and NUDT17 expression revealed that they were normally distributed (data not shown), so TCGA cohort was divided into low and high expression groups according to the median expression level. As a result, higher NUDT5 $(\mathrm{P}<0.0001)$ and NUDT17 $(\mathrm{P}<0.0001)$ expression was associated with better prognosis for OS, whereas high levels of NUDT9P1 $(\mathrm{P}=0.151)$ and NUDT16 $(\mathrm{P}=0.153)$ expression was not associated with OS prognosis (Fig. 1).

In multivariate logistic regression analysis of factors that could affect the expression of NUDT5, NUDT9P1, NUDT16 and NUDT17, tumor grade was significantly associated with NUDT5 $(\mathrm{P}=0.006)$ and NUDT17 $(\mathrm{P}=0.002)$ expression, while tumor stage was also significantly associated with NUDT5 $(\mathrm{P}=0.001)$ and NUDT17 $(\mathrm{P}=0.007)$ expression (Table III).

To understand the different expression of NUDT family between patients with ccRCC and normal population further, the present study analyzed the expression of NUDT family in 70 couples of paired patients. If deviations in NUDT expression between couples fitted a normal distribution, paired student t-tests were performed; if not, Wilcoxon signed-rank test was performed. Using a paired Student's t-test, the expression of NUDT3, NUDT4, NUDT6, NUDY7, NUDT9SP1, NUDT12, NUDT13, NUDT15, NUDT16 and NUDT16SP1 was found to be significantly different between patients with ccRCC and paired healthy individuals, whereas differences in the expression of NUDT17 was not statistically significant. Using a Wilcoxon signed-rank test, expression of NUDT1, NUDT8, NUDT9, NUDT10, NUDT11, NUDT16L1, NUDT18 and NUDT21 were significantly different between patients with ccRCC and paired healthy individuals, whereas expression of NUDT2, NUDT5, NUDt14, NUDT19 and NUDT22 did not differ significantly (Table IV).

NUDT5 expressions were prognostic factors for $O S$ in the FUSCC cohort. NUDT5, NUDT9P1, NUDT16 and NUDT17 expression was validated in the FUSCC cohort. This cohort was then divided into low- and high-expression groups according to the median expression level. As the expression level of genes was based on the relative values of PCR results, patients were grouped by $\Delta-\mathrm{Ct}$ (cycle threshold). $\Delta-\mathrm{Ct}=\mathrm{Ct}$ (target genes)-Ct (reference genes). The median $\Delta$-Ct value of NUDT5, NUDT9P1, NUDT16 and NUDT17 were 8.29, 3.90, 7.32 and 4.67, respectively. As a result, low NUDT5 expression was associated with poor OS (log-rank test, $\mathrm{P}=0.0116$ ), 
Table II. Univariate and multivariate Cox Proportional Hazards analysis of integrin expression and overall survival for patients with clear cell renal cell carcinoma in The Cancer Genome Atlas cohort.

\begin{tabular}{|c|c|c|c|c|c|c|}
\hline \multirow[b]{2}{*}{ Variables } & \multicolumn{3}{|c|}{ Univariate } & \multicolumn{3}{|c|}{ Multivariate } \\
\hline & HR & $95 \% \mathrm{CI}$ & $\mathrm{P}$-value & HR & $95 \% \mathrm{CI}$ & P-value \\
\hline Age & 1.028 & $1.015-1.042$ & $<0.001$ & 1.036 & $1.020-1.053$ & $<0.001$ \\
\hline Sex & 1.073 & $0.781-1.473$ & 0.665 & & & \\
\hline $\mathrm{T}$ & 1.964 & $1.658-2.325$ & $<0.001$ & & & \\
\hline $\mathrm{N}$ & 2.799 & $1.486-5.274$ & 0.001 & & & \\
\hline M & 4.448 & $3.221-6.141$ & $<0.001$ & & & \\
\hline Stage & 1.944 & $1.695-2.229$ & $<0.001$ & 1.603 & $1.317-1.949$ & $<0.001$ \\
\hline Grade & 2.350 & $1.899-2.908$ & $<0.001$ & 1.230 & $0.920-1.644$ & 0.162 \\
\hline $\mathrm{Hb}$ & 0.584 & $0.415-0.823$ & 0.002 & 0.915 & $0.624-1.342$ & 0.651 \\
\hline WBC & 0.652 & $0.471-0.902$ & 0.010 & 1.014 & $0.694-1.483$ & 0.942 \\
\hline PLT & 1.702 & $1.145-2.529$ & 0.008 & 1.086 & $0.748-1.579$ & 0.664 \\
\hline Tumor size & 1.174 & $0.946-1.459$ & 0.146 & & & \\
\hline Laterality & 0.669 & $0.491-0.913$ & 0.011 & 0.664 & $0.467-0.944$ & 0.023 \\
\hline NUDT1 & 1.629 & $1.346-1.971$ & $<0.001$ & 0.942 & $0.661-1.341$ & 0.740 \\
\hline NUDT2 & 0.779 & $0.596-1.018$ & 0.068 & & & \\
\hline NUDT3 & 2.089 & $1.215-3.593$ & 0.008 & 0.739 & $0.401-1.361$ & 0.332 \\
\hline NUDT4 & 0.706 & $0.538-0.925$ & 0.011 & 0.849 & $0.614-1.175$ & 0.324 \\
\hline NUDT5 & 2.165 & $1.684-2.783$ & $<0.001$ & 1.676 & $1.097-2.559$ & 0.017 \\
\hline NUDT6 & 0.608 & $0.461-0.801$ & $<0.001$ & 0.947 & $0.648-1.383$ & 0.778 \\
\hline NUDT7 & 0.716 & $0.576-0.889$ & 0.003 & 1.032 & $0.730-1.457$ & 0.859 \\
\hline NUDT8 & 1.113 & $0.953-1.299$ & 0.174 & & & \\
\hline NUDT9 & 0.971 & $0.639-1.474$ & 0.888 & & & \\
\hline NUDT9P1 & 1.274 & $1.011-1.606$ & 0.040 & 1.512 & $1.143-2.000$ & 0.004 \\
\hline NUDT10 & 1.203 & $1.074-1.347$ & 0.001 & 1.180 & 0.987-1.412 & 0.069 \\
\hline NUDT11 & 1.347 & $1.208-1.503$ & $<0.001$ & 0.962 & $0.796-1.162$ & 0.684 \\
\hline NUDT12 & 0.661 & $0.542-0.805$ & $<0.001$ & 0.781 & $0.600-1.017$ & 0.066 \\
\hline NUDT13 & 0.991 & $0.809-1.214$ & 0.931 & & & \\
\hline NUDT14 & 0.934 & $0.778-1.121$ & 0.464 & & & \\
\hline NUDT15 & 0.742 & 0.496-1.107 & 0.144 & & & \\
\hline NUDT16 & 0.677 & 0.499-0.919 & 0.012 & 0.692 & $0.486-0.985$ & 0.041 \\
\hline NUDT16P1 & 0.947 & $0.792-1.133$ & 0.554 & & & \\
\hline NUDT16L1 & 1.096 & $0.813-1.478$ & 0.546 & & & \\
\hline NUDT17 & 1.583 & $1.341-1.870$ & $<0.001$ & 1.375 & $1.092-1.732$ & 0.007 \\
\hline NUDT18 & 0.975 & $0.788-1.206$ & 0.817 & & & \\
\hline NUDT19 & 2.026 & $1.372-2.991$ & $<0.001$ & & & \\
\hline NUDT21 & 0.700 & $0.519-0.946$ & 0.020 & 1.113 & $0.682-1.815$ & 0.668 \\
\hline NUDT22 & 1.424 & $1.127-1.800$ & 0.003 & 1.090 & $0.686-1.730$ & 0.716 \\
\hline
\end{tabular}

HR, hazard ratio; CI, confidence interval; T, Tumor; N, Node; M, Metastasis; Hb, hemoglobin; WBC, white blood cells; PLT, platelets; NUDT1, nudix hydroxylase 1; NUDTP1, NUDT9 pseudogene 1; NUDT16L1, NUDT16-like 1.

although the level of NUDT9P1 (log-rank test, $\mathrm{P}=0.5915)$, NUDT16 (log-rank test, $\mathrm{P}=0.6814$ ) and NUDT17 (log-rank test, $\mathrm{P}=0.2968$ ) expression was not associated with $\mathrm{OS}$. The Kaplan-Meier curves are shown in Fig. 2.

To understand the factors that may affect the expression of NUDT5, NUDT9P1, NUDT16 and NUDT17 in the FUSCC cohort further, multivariate logistic regression analysis with the same parameters including age, stage, grade, hemoglobin level; white blood cells level and platelets level was performed.
In the FUSCC cohort, tumor grade was significantly associated with the NUDT5 expression level $(\mathrm{P}=0.016)$ expression, whereas other parameters were not significantly associated with the expression of NUDTs (Table V).

\section{Discussion}

To the best of our knowledge, the present study demonstrates that expression of the NUDT family of genes is associated 
Table III. Multivariate logistic regression analysis of factors that might affect the expression of NUDT5, NUDT9P1, NUDT16 and NUDT17 in The Cancer Genome Atlas cohort with clear cell renal cell carcinoma.

\begin{tabular}{|c|c|c|c|c|c|c|c|c|c|c|c|c|}
\hline \multirow[b]{2}{*}{ Variables } & \multicolumn{3}{|c|}{ NUDT5 } & \multicolumn{3}{|c|}{ NUDT9P1 } & \multicolumn{3}{|c|}{ NUDT16 } & \multicolumn{3}{|c|}{ NUDT17 } \\
\hline & OR & $95 \% \mathrm{CI}$ & P-value & OR & $95 \% \mathrm{CI}$ & P-value & OR & $95 \% \mathrm{CI}$ & P-value & OR & $95 \% \mathrm{CI}$ & P-value \\
\hline Age & 1.009 & $0.992-1.026$ & 0.288 & 0.984 & $0.968-1.000$ & 0.055 & 1.008 & $0.991-1.023$ & 0.374 & 0.994 & $0.978-1.011$ & 0.507 \\
\hline Stage & 1.409 & $1.148-1.732$ & 0.001 & 1.155 & $0.947-1.409$ & 0.155 & 0.926 & $0.760-1.128$ & 0.446 & 1.318 & $1.078-1.613$ & 0.007 \\
\hline Grade & 1.568 & $1.138-2.160$ & 0.006 & 0.800 & $0.590-1.085$ & 0.152 & 1.029 & $0.761-1.390$ & 0.855 & 1.637 & $1.197-2.239$ & 0.002 \\
\hline $\mathrm{Hb}$ & 0.898 & $0.588-1.370$ & 0.617 & 0.734 & $0.489-1.103$ & 0.137 & 1.171 & $0.783-1.752$ & 0.442 & 0.961 & $0.633-1.459$ & 0.852 \\
\hline WBC & 0.937 & $0.604-1.453$ & 0.771 & 0.804 & $0.529-1.222$ & 0.307 & 1.302 & $0.859-1.973$ & 0.213 & 0.977 & $0.635-1.502$ & 0.916 \\
\hline PLT & 1.563 & $0.013-0.359$ & 0.085 & 1.221 & $0.765-1.949$ & 0.403 & 0.942 & $0.593-1.496$ & 0.799 & 0.742 & $0.458-1.201$ & 0.226 \\
\hline
\end{tabular}

NUDT5, nudix hydroxylase 5; NUDT9P1, NUDT9 psuedogene 1; OR, odds ratio; CI, confidence interval; Hb, hemoglobin; WBC, white blood cells; PLT, platelets.

A

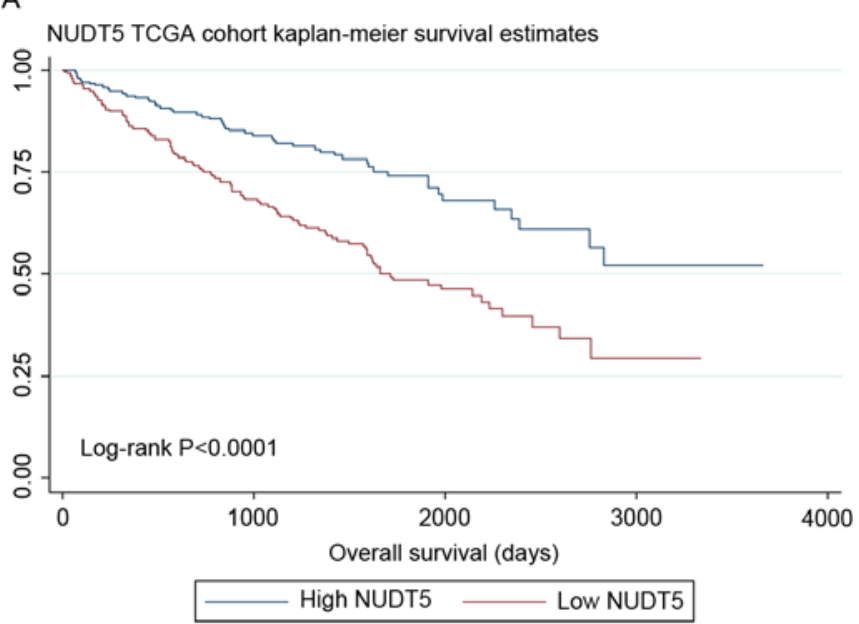

C

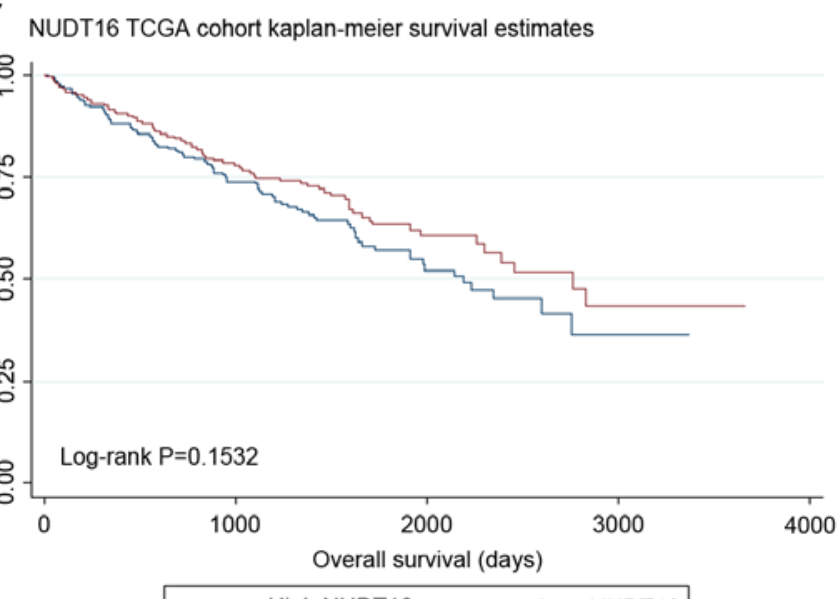

B

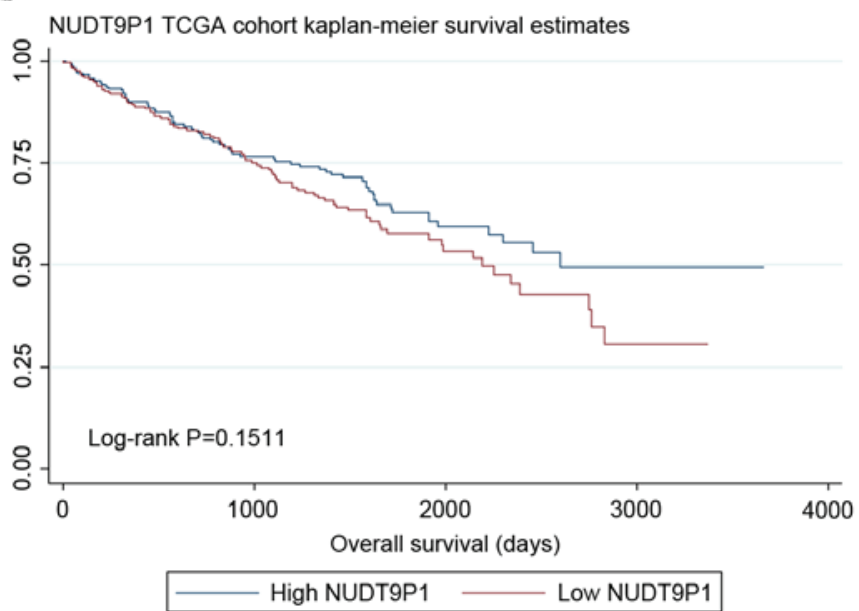

D

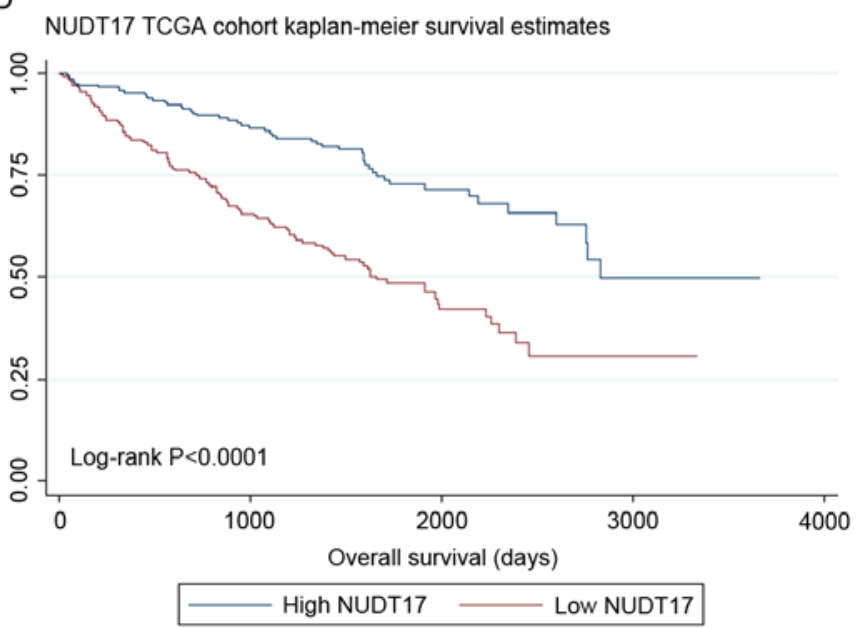

Figure 1. Kaplan-Meier plots of survival in TCGA cohort are shown according to NUDT5, NUDT9P1, NUDT16 and NUDT17 expression. (A) Kaplan-Meier estimates of OS are shown according to the expression level of NUDT5. (B) Kaplan-Meier estimates of OS are shown according to the expression level of NUDT9P1. (C) Kaplan-Meier estimates of OS are shown according to the expression level of NUDT16. (D) Kaplan-Meier estimates of OS are shown according to the expression level of NUDT17. TCGA, The Cancer Genome Atlas; NUDT5, nudix hydroxylase 5; NUDT9P1, NUDT9 psuedogene 1; OS, overall survival.

with the OS of patients with ccRCC. Members of this family, particularly NUDT5, NUDT9P1, NUDT16 and NUDT17, may be independent prognostic factors for OS in patients with ccRCC.
The present study demonstrates that the NUDT family may have important roles in suppressing the progression of ccRCC. NUDT5, NUDT9P1, NUDT16 and NUDT17 expression were independent prognostic factors for OS in patients 
Table IV. Expression of NUDT family genes in 70 patients and paired healthy individuals in The Cancer Genome Atlas cohort.

\begin{tabular}{lrll}
\hline Variables & \multicolumn{1}{c}{ P-value } & \multicolumn{1}{c}{ Statistical test } & \multicolumn{1}{c}{$95 \%$ CI } \\
\hline NUDT9P1 & $<0.001$ & Paired Student t-test & $0.364-0.677$ \\
NUDT16L1 & $<0.001$ & Wilcoxon rank-sum test & \\
NUDT12 & $<0.001$ & Paired Student t-test & $0.467-0.818$ \\
NUDT10 & $<0.001$ & Wilcoxon rank-sum test & \\
NUDT17 & 0.117 & Paired Student t-test & $-0.490-0.056$ \\
NUDT14 & 0.301 & Wilcoxon rank-sum test & \\
NUDT15 & $<0.001$ & Paired Student t-test & $0.110-0.338$ \\
NUDT18 & $<0.001$ & Wilcoxon rank-sum test & \\
NUDT19 & 0.859 & Wilcoxon rank-sum test & \\
NUDT11 & $<0.001$ & Wilcoxon rank-sum test & \\
NUDT13 & $<0.001$ & Paired Student t-test & $0.199-0.531$ \\
NUDT1 & $<0.001$ & Wilcoxon rank-sum test & \\
NUDT2 & 0.850 & Wilcoxon rank-sum test & \\
NUDT3 & 0.001 & Paired Student t-test & $0.0742-0.279$ \\
NUDT4 & $<0.001$ & Paired Student t-test & $1.638-2.140$ \\
NUDT5 & 0.149 & Wilcoxon rank-sum test & \\
NUDT6 & $<0.001$ & Paired Student t-test & $0.951-1.344$ \\
NUDT7 & $<0.001$ & Paired Student t-test & $0.667-0.982$ \\
NUDT9 & $<0.001$ & Wilcoxon rank-sum test & \\
NUDT8 & 0.002 & Wilcoxon rank-sum test & \\
NUDT22 & 0.160 & Wilcoxon rank-sum test & \\
NUDT21 & $<0.001$ & Wilcoxon rank-sum test & \\
NUDT16P1 & $<0.001$ & Paired Student t-test & $0.322-0.633$ \\
NUDT16 & $<0.001$ & Paired Student t-test & $0.559-0.821$ \\
\hline & & & \\
NUDT5, & & &
\end{tabular}

NUDT5, nudix hydroxylase 5; NUDT9P1, NUDT9 psuedogene 1; NUDT16L1, NUDT16-like 1; CI, confidence interval.

with ccRCC. Reduced expression of NUDT5 and NUDT17 was associated with poor prognosis and decreased OS time. Expression of NUDT5 is closely associated with the prognosis of patients with ccRCC. Additionally, Fuhrman grade and TNM stage were significantly associated with NUDT5 and NUDT17 expression. However, upon statistical analysis of 70 paired patients with ccRCC and healthy individuals, there was no significant difference in expression of NUDT5 and NUDT17. This may be because: i) The number of patients included in the paired study was not large enough; and ii) in analysis of NUDT5, the Wilcoxon signed-rank test was used, which has a low power and thus may affect the outcome of the analysis. However, in patients in TCGA database, NUDT5 and NUDT17 remained good indicators of prognosis. To further verify the accuracy of NUDT as a ccRCC prognostic marker, FUSCC patient specimens were tested. Low NUDT5 expression was associated with OS rates in the FUSCC cohort. Even though no statistical difference existed between low and high NUDT17 expression groups in the FUSCC cohort, NUDT5 remains a good prognostic indicator, due to demographic difference between these two studies. All patients in the present study were Asian, while the majority of the patients in the TCGA cohort were Caucasian or of African descent.
The human genome has 24 NUDT hydrolase genes and at least 5 pseudogenes (18). NUDT genes are associated with metabolic reprogramming and mutagenesis. Previous studies have partially revealed their functions, even though the role they serve in tumorigenesis is poorly understood $(16,17)$.

NUDT5 is an antimutator candidate; this protein was originally characterized as an ADP sugar hydrolase, which corresponds to the high- $\mathrm{K}_{\mathrm{m}}$ ADP Ribose-II isolated from tissues. ADP Ribose is a member of a family of proteins involved in a number of cellular processes such as DNA repair, genomic stability and programmed cell death $(19,20)$. In experiments in vitro, NUDT5 suppressed the increased mutation rate of cancer cells and may act in concert with NUDT1 or NUDT15 in antimutagenesis $(19,20)$. NUDT5 may also prevent transcriptional errors and mistranslation. Prior studies (19-21) also found that lowered NUDT5 expression led to cell cycle inhibition in HeLa cells (21). Further studies indicated that the NUDT5 protein may have notable roles in regulating the G1-S transition in mammalian cells (22-24).

Nudix hydrolase 9 pseudogene 1 (NUDT9P1) is located in the 5-HT receptor 7, adenylate cyclase-coupled (HTR7) gene, which is associated with the response to iloperidone. However, the role of NUDT9P1 in healthy or tumor cells remains unknown (25).

NUDT16 is a 'housecleaning' enzyme that removes inosine diphosphate from the nucleotide pool. Studies have revealed that NUDT16 forms a dimer, which generates a positively charged trench to accommodate substrate binding (26). NUDT16 may be involved in regulating ribosome biogenesis by altering the stability of U8 small nucleolar RNA and other guide RNAs $(26,27)$. Studies have revealed that NUDT16 may interact with a nuclear protein phosphatase, possibly in a complex with small nuclear riboprotein components $(28,29)$.

At the time of writing, NUDT17 remains an uncharacterized protein, with no known function. NUDT17 may be bi-functional and possess mRNA de-capping activity in cells, in addition to its reported activities on nucleotide containing molecules (16).

Little is known about the NUDT family of genes in the field of oncology. NUDT1 and NUDT15 are expressed in RAS-dependent types of cancer (30,31). Loss of NUDT1 function impaired growth of KRAS proto-oncogene, GTPase-positive tumor cells. NUDT1 overexpression mitigated sensitivity towards certain experimental small molecules, including the NUDT1 inhibitor SCH51344 (30-32). However, the association between NUDT family and tumorigenesis were not clear and studies about their role in renal cancer are rare (30-32).

The present study confirmed the role of the NUDT family of genes in patients with ccRCC, identifying NUDT5 may inform on patient prognosis. Limitations of the present study are: i) All of the patients that were included in the present study were from Fudan University Shanghai Cancer Center with excellent follow-up, and patients from other centers were not included; ii) all patient tissue specimens in the present study came from patients who suitable to surgery so it is possible that the results will not apply to people who were not suitable for surgery; and iii) the number of patients who participated in the study was low.

The present study indicated the presence of an association between ccRCC outcome and NUDT gene family expression; 
Table V. Multivariate logistic regression analysis of factors that might affect the expression of NUDT5, NUDT9P1, NUDT16 and NUDT17 in the Fudan University Shanghai Cancer Center cohort with clear cell renal cell carcinoma.

\begin{tabular}{|c|c|c|c|c|c|c|c|c|c|c|c|c|}
\hline \multirow[b]{2}{*}{ Variables } & \multicolumn{3}{|c|}{ NUDT5 } & \multicolumn{3}{|c|}{ NUDT9P1 } & \multicolumn{3}{|c|}{ NUDT16 } & \multicolumn{3}{|c|}{ NUDT17 } \\
\hline & OR & $95 \% \mathrm{CI}$ & P-value & OR & $95 \% \mathrm{CI}$ & P-value & OR & $95 \% \mathrm{CI}$ & P-value & OR & $95 \% \mathrm{CI}$ & P-value \\
\hline Age & 0.992 & $0.959-1.027$ & 0.667 & 0.984 & $0.950-1.018$ & 0.355 & 0.978 & 0.94 & 0.225 & 0.992 & 0.958 & 0.659 \\
\hline Stage & 0.663 & $0.409-1.077$ & 0.097 & 1.380 & $0.868-2.197$ & 174 & 1.000 & 0.616 & 98 & 1.539 & $0.961-2.462$ & 0.073 \\
\hline Grade & 2.132 & $1.150-3.951$ & 0.016 & 0.770 & $0.437-1.356$ & 0.366 & 0.789 & $0.438-1.421$ & 0.430 & 1.400 & $0.794-2.470$ & 0.245 \\
\hline $\mathrm{Hb}$ & 0.984 & $0.958-1.011$ & 0.243 & 1.023 & $0.995-1.051$ & 0.104 & 1.011 & $0.984-1.038$ & 0.420 & 1.018 & $0.991-1.046$ & 0.198 \\
\hline WBC & 0.995 & $0.987-1.003$ & 0.222 & 0.999 & $0.993-1.005$ & 0.723 & 0.993 & $0.981-1.005$ & 0.275 & 0.997 & $0.992-1.003$ & 0.412 \\
\hline PLT & 1.002 & $0.996-1.007$ & 0.561 & 0.998 & $0.992-1.003$ & 0.477 & 1.001 & $0.995-1.007$ & 0.672 & 1.004 & $0.998-1.009$ & 0.192 \\
\hline
\end{tabular}

NUDT5, nudix hydroxylase 5; NUDT9P1, NUDT9 psuedogene 1; OR, odds ratio; CI, confidence interval; Hb, hemoglobin; WBC, white blood cells; PLT, platelets.

A

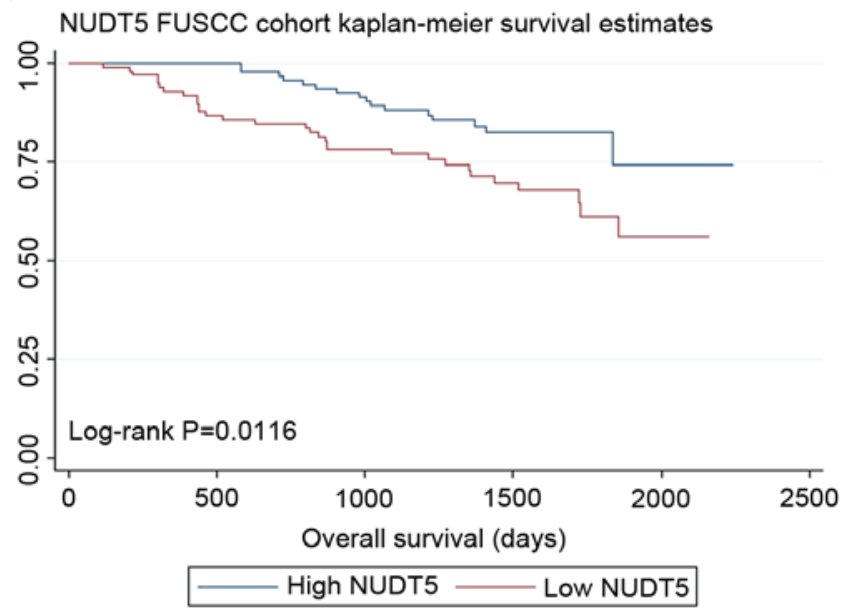

C

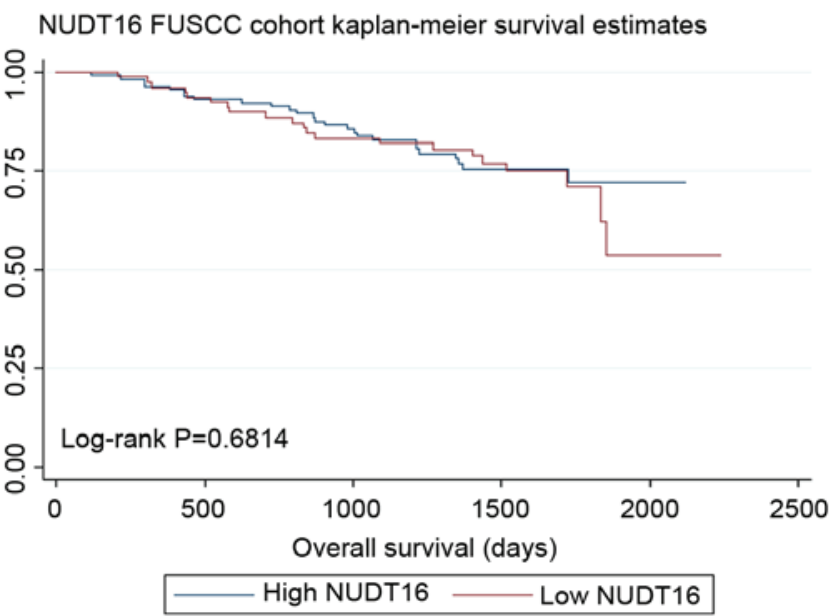

B

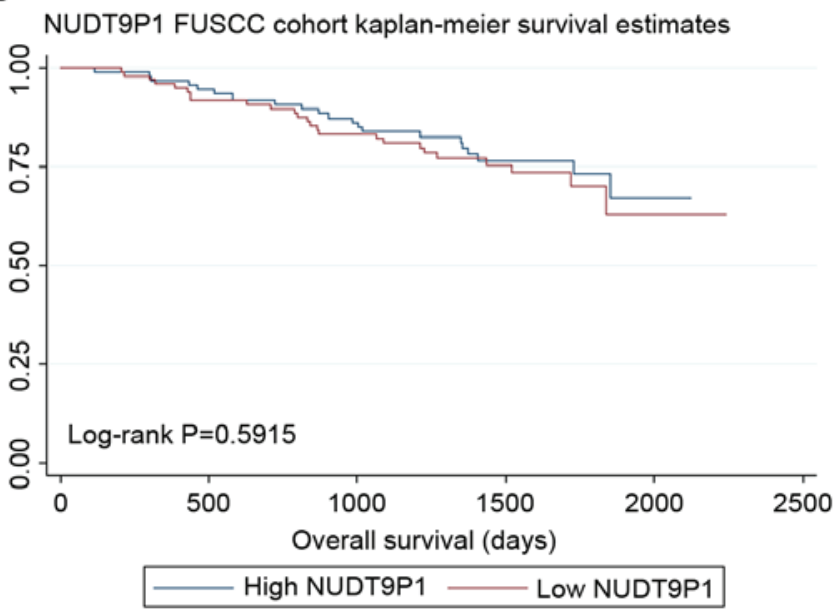

D

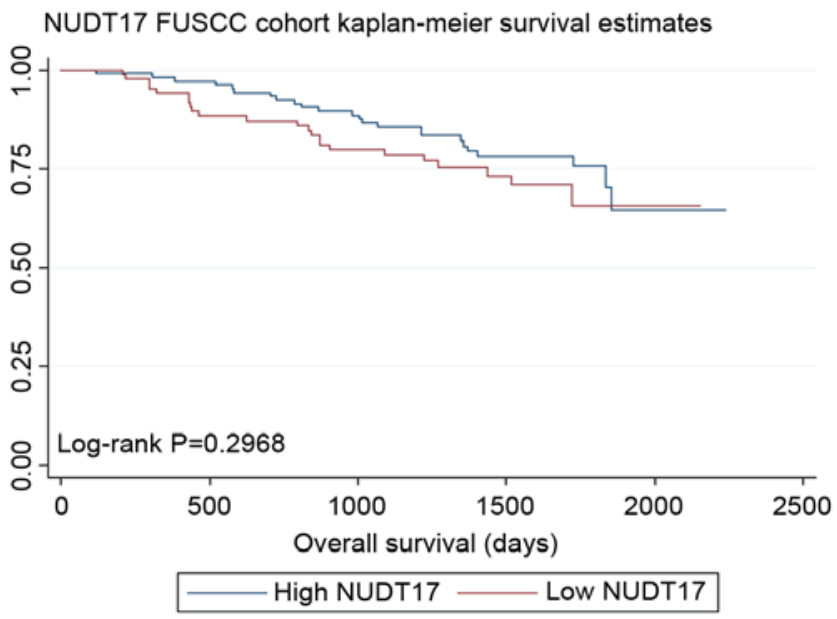

Figure 2. Kaplan-Meier plots of survival in the FUSCC cohort are shown according to NUDT5, NUDT9P1, NUDT16 and NUDT17 expression. (A) Kaplan-Meier estimates of OS are shown according to the expression level of NUDT5. (B) Kaplan-Meier estimates of OS are shown according to the expression level of NUDT9P1. (C) Kaplan-Meier estimates of OS are shown according to the expression level of NUDT16. (D) Kaplan-Meier estimates of OS are shown according to the expression level of NUDT17. FUSCC, Fudan University Shanghai Cancer Center; NUDT5, nudix hydroxylase 5; NUDT9P1, NUDT9 psuedogene 1; OS, overall survival.

however, the underlying mechanism has yet to be elucidated. The present study may have revealed novel ccRCC biomarkers or therapeutic targets; as such, further study is urged.
NUDT5 expression was identified as an independent prognostic factor for OS time of ccRCC in the present study: Low NUDT5 expression was associated with low OS time and 
tumor grade was significantly associated with NUDT5 expression. NUDT5 could therefore act as a tool to reveal further prognostic genes in ccRCC.

\section{Acknowledgements}

The present study was supported in part by the grants for International Cooperation and Exchange of Science and Technology Commission of Shanghai Municipality (no. 12410709300), from the Guide Project of Science and Technology Commission of Shanghai Municipality (no. 124119a7300), and from the Outstanding Young Talent Training Plan of Shanghai Municipal Commission of Health and Family Planning (no. XYQ2013102).

\section{References}

1. Ljungberg B, Campbell SC, Choi HY, Jacqmin D, Lee JE, Weikert S and Kiemeney LA: The epidemiology of renal cell carcinoma. Eur Urol 60: 615-621, 2011.

2. Ridge CA, Pua BB and Madoff DC: Epidemiology and staging of renal cell carcinoma. Semin Intervent Radiol 31: 3-8, 2014.

3. Shuch B, Ricketts CJ, Vocke CD, Komiya T, Middelton LA, Kauffman EC, Merino MJ, Metwalli AR, Dennis P and Linehan WM: Germline PTEN mutation Cowden syndrome: An underappreciated form of hereditary kidney cancer. J Urol 190: 1990-1998, 2013.

4. Chow WH, Dong LM and Devesa SS: Epidemiology and risk factors for kidney cancer. Nat Rev Urol 7: 245-257, 2010.

5. Ljungberg B, Cowan NC, Hanbury DC, Hora M, Kuczyk MA, Merseburger AS, Patard JJ, Mulders PF and Sinescu IC; European Association of Urology Guideline Group: EAU guidelines on renal cell carcinoma: The 2010 update. Eur Urol 58: 398-406, 2010.

6. Siegel RL, Miller KD and Jemal A: Cancer statistics, 2016. CA Cancer J Clin 66: 7-30, 2016.

7. Maher ER: Genomics and epigenomics of renal cell carcinoma Semin Cancer Biol 23: 10-17, 2013.

8. Linehan WM, Srinivasan R and Schmidt LS: The genetic basis of kidney cancer: A metabolic disease. Nat Rev Urol 7: 277-285, 2010.

9. Bessman MJ, Frick DN and O'Handley SF: The MutT proteins or 'Nudix' hydrolases, a family of versatile, widely distributed, 'housecleaning' enzymes. J Biol Chem 271: 25059-25062, 1996.

10. Ito R, Hayakawa H, Sekiguchi M and Ishibashi T: Multiple enzyme activities of Escherichia coli MutT protein for sanitization of DNA and RNA precursor pools. Biochemistry 44: 6670-6674, 2005.

11. Xu W, Jones CR, Dunn CA and Bessman MJ: Gene ytkD of Bacillus subtilis encodes an atypical nucleoside triphosphatase member of the Nudix hydrolase superfamily. J Bacteriol 186: 8380-8384, 2004.

12. Fisher DI, Cartwright JL, Harashima H, Kamiya $\mathrm{H}$ and McLennan AG: Characterization of a nudix hydrolase from Deinococcus radiodurans with a marked specificity for (deoxy) ribonucleoside 5'-diphosphates. BMC Biochem 5: 7, 2004.

13. Safrany ST, Caffrey JJ, Yang X, Bembenek ME, Moyer MB, Burkhart WA and Shears SB: A novel context for the 'MutT module, a guardian of cell integrity, in a diphosphoinositol polyphosphate phosphohydrolase. EMBO J 17: 6599-6607, 1998.

14. Fujikawa K, Kamiya H, Yakushiji H, Fujii Y, Nakabeppu Y and Kasai H: The oxidized forms of dATP are substrates for the human MutT homologue, the hMTH1 protein. J Biol Chem 274: 18201-18205, 1999.

15. Kamiya H, Yakushiji H, Dugué L, Tanimoto M, Pochet S, Nakabeppu Y and Harashima H: Probing the substrate recognition mechanism of the human MTH1 protein by nucleotide analogs. J Mol Biol 336: 843-850, 2004.
16. Caffrey JJ, Safrany ST, Yang X and Shears SB: Discovery of molecular and catalytic diversity among human diphosphoinositol-polyphosphate phosphohydrolases. An expanding Nudt family. J Biol Chem 275: 12730-12736, 2000.

17. McLennan AG, Cartwright JL and Gasmi L: The human NUDT family of nucleotide hydrolases. Enzymes of diverse substrate specificity. Adv Exp Med Biol 486: 115-118, 2000.

18. Kamiya H, Cadena-Amaro C, Dugué L, Yakushiji H, Minakawa N, Matsuda A, Pochet S, Nakabeppu Y and Harashima H: Recognition of nucleotide analogs containing the 7,8-dihydro-8-oxo structure by the human MTH1 protein. J Biochem 140: 843-849, 2006.

19. Gasmi L, Cartwright JL and McLennan AG: Cloning, expression and characterization of YSA1H, a human adenosine 5'-diphosphosugar pyrophosphatase possessing a MutT motif. Biochem J 344: 331-337, 1999 .

20. Yang H, Slupska MM, Wei YF, Tai JH, Luther WM, Xia YR, Shih DM, Chiang JH, Baikalov C, Fitz-Gibbon S, et al: Cloning and characterization of a new member of the Nudix hydrolases from human and mouse. J Biol Chem 275: 8844-8853, 2000.

21. Zhang LQ, Dai DP, Gan W, Takagi Y, Hayakawa H, Sekiguchi M and Cai JP: Lowered nudix type 5 (NUDT5) expression leads to cell cycle retardation in HeLa cells. Mol Cell Biochem 363: 377-384, 2012.

22. Fujiwara S, Sawada K and Amisaki T: Molecular dynamics study on conformational differences between dGMP and 8-oxo-dGMP: Effects of metal ions. J Mol Graph Model 51: 158-167, 2014.

23. Arimori T, Tamaoki H, Nakamura T, Kamiya H, Ikemizu S, Takagi Y, Ishibashi T, Harashima H, Sekiguchi M and Yamagata Y: Diverse substrate recognition and hydrolysis mechanisms of human NUDT5. Nucleic Acids Res 39: 8972-8983, 2011.

24. Zha M, Guo Q, Zhang Y, Yu B, Ou Y, Zhong C and Ding J: Molecular mechanism of ADP-ribose hydrolysis by human NUDT5 from structural and kinetic studies. J Mol Biol 379: 568-578, 2008.

25. Lavedan C, Licamele L, Volpi S, Hamilton J, Heaton C, Mack K, Lannan R, Thompson A, Wolfgang CD and Polymeropoulos MH: Association of the NPAS3 gene and five other loci with response to the antipsychotic iloperidone identified in a whole genome association study. Mol Psychiatry 14: 804-819, 2009.

26. Lu G, Zhang J, Li Y, Li Z, Zhang N, Xu X, Wang T, Guan Z, Gao GF and Yan J: hNUDT16: A universal decapping enzyme for small nucleolar RNA and cytoplasmic mRNA. Protein Cell 2: 64-73, 2011

27. Li Y, Song M and Kiledjian M: Differential utilization of decapping enzymes in mammalian mRNA decay pathways. RNA 17: 419-428, 2011.

28. Trésaugues L, Lundbäck T, Welin M, Flodin S, Nyman T, Silvander C, Gräslund S and Nordlund P: Structural basis for the specificity of human NUDT16 and its regulation by inosine monophosphate. PLoS One 10: e0131507, 2015.

29. Abolhassani N, Iyama T, Tsuchimoto D, Sakumi K, Ohno M, Behmanesh M and Nakabeppu Y: NUDT16 and ITPA play a dual protective role in maintaining chromosome stability and cell growth by eliminating dIDP/IDP and dITP/ITP from nucleotide pools in mammals. Nucleic Acids Res 38: 2891-2903, 2010.

30. Dallosso AR, Dolwani S, Jones N, Jones S, Colley J, Maynard J, Idziaszczyk S, Humphreys V, Arnold J, Donaldson A, et al: Inherited predisposition to colorectal adenomas caused by multiple rare alleles of MUTYH but not OGG1, NUDT1, NTH1 or NEIL 1, 2 or 3. Gut 57: 1252-1255, 2008.

31. Cho WC, Chow AS and Au JS: MiR-145 inhibits cell proliferation of human lung adenocarcinoma by targeting EGFR and NUDT1. RNA Biol 8: 125-131, 2011.

32. Garre P, Briceño V, Xicola RM, Doyle BJ, de la Hoya M, Sanz J, Llovet P, Pescador P, Puente J, Díaz-Rubio E, et al: Analysis of the oxidative damage repair genes NUDT1, OGG1, and MUTYH in patients from mismatch repair proficient HNPCC families (MSS-HNPCC). Clin Cancer Res 17: 1701-1712, 2011. 